\title{
Multihop Transmission Opportunity in Wireless Multihop Networks
}

\author{
Chansu Yu and Tianning Shen \\ Department of Electrical and \\ Computer Engineering \\ Cleveland State University \\ Cleveland, Ohio 44115 \\ Email: \{c.yu91,t.shen99\}@csuohio.edu
}

\author{
Kang G. Shin \\ Department of Electrical Engineering \\ and Computer Science \\ University of Michigan \\ Ann Arbor, MI 48109 \\ Email: kgshin@eecs.umich.edu
}

\author{
Jeong-Yoon Lee and Young-Joo Suh \\ Department of Computer \\ Science and Engineering \\ Pohang Univ. of Sci. and Tech. (POSTECH) \\ Pohang, 790-784, Republic of Korea \\ Email: \{jylee9, yjsuh\}@ postech.ac.kr
}

\begin{abstract}
Wireless multihop communication is becoming more important due to the increasing popularity of wireless sensor networks, wireless mesh networks, and mobile social networks. They are distinguished from conventional multihop networks in terms of scale, traffic intensity and/or node density. Being readilyavailable in most of 802.11 radios, multirate facility appears to be useful to address some of these issues and is particularly helpful in high-density scenarios where inter-node distance is short, demanding a prudent multirate adaptation algorithm. However, communication at high bit rates mandates a large number of hops for a given node pair and thus, can easily be depreciated as per-hop overhead at several layers of network protocol is aggregated over the increased number of hops. This paper presents a novel multihop, multirate adaptation mechanism, called Multihop Transmission OPportunity (MTOP), that allows a frame to be forwarded a number of hops consecutively but reduces the MAC-layer overhead between hops. This seemingly collision-prone multihop forwarding is proven to be safe via analysis and USRP/GNU Radio-based experiment. The idea of MTOP is in clear contrast to, but not mutually exclusive with, the conventional opportunistic transmission mechanism, referred to as TXOP, where a node transmits multiple frames back-to-back when it gets an opportunity. We conducted an extensive simulation study via ns-2, demonstrating the performance advantage of MTOP under a wide range of network scenarios.
\end{abstract}

\section{INTRODUCTION}

Wireless LANs (WLANs) based on IEEE 802.11 standard [1] are in great popularity in public as well as in residential areas. However, WLAN hardware and the underlying 802.11 standard have been originally developed for singlehop wireless communications between an access point (AP) and end clients, which may not be appropriate for multihop communications abundant in wireless sensor networks (WSN), the backhaul of wireless mesh networks (WMN) [2], [3], and mobile social networks (MSN) [4], [5].

These multihop networks exhibit characteristics that deviate significantly from the traditional ad hoc networks in terms of scale, traffic intensity and node density. For example, MSN scenarios typically envisaged around crowd spots, where the number of nodes within range could be hundreds or thousands [6]. A similar high density scenario has been already observed in 802.11 deployments and WMNs in US cities such that the number of other APs within interference range of an AP is as many as 85 [7]. These facts imply a shorter inter-node distance, opening opportunities to utilize it.

Multirate capability seems to be a promising radio facility that can effectively exploit the short inter-node distance in high-density networks owing to more chances to use high rates considering the rate-distance tradeoff. However, it is important to observe that performance does not improve linearly as bit rate increases. This is due to the rate-independent overhead at the PHY and MAC layers, which are imposed by 802.11 standards [1]. Moreover, this overhead becomes a major part as rate increases because the transmission time of the payload decreases proportionally. In $802.11 \mathrm{~b}$, per-frame PHY-layer overhead for a 512-byte frame is $4.5 \%$ at $1 \mathrm{Mbps}$ but is increased to $34 \%$ at $11 \mathrm{Mbps}$. More seriously, MAClayer overhead for a 512-byte frame is $15 \sim 71 \%$ of total communication time at 1Mbps, which amounts to 57 95\% at $11 \mathrm{Mbps}$ (see Section II-B).

Opportunistic transmission protocols (TXOP) [8]-[11] have been proposed to alleviate the MAC-layer overhead by allowing a node to transmit multiple frames back-to-back when it gets a chance (say, $0 \rightarrow 1,0 \rightarrow 2,0 \rightarrow 3$, etc.). Figs. 1(a) and 1 (b) show the communication sequence in 802.11 and TXOP, respectively. In TXOP, node 0 is allowed to transmit multiple frames with the inter-frame gap of SIFS only (10 $\mu \mathrm{s})$ reducing the MAC overhead. However, TXOP is effective only in the context of WLANs and may defeat the usual expectation in multihop networks because (i) a node may not have multiple frames to transmit back-to-back although it is given an opportunity, (ii) it can easily overload intermediate nodes in a multihop chain when a predecessor grabs more transmission opportunities than its successors and, (iii) it does not resolve "multihop" performance anomaly problem described in Section II-D.

\section{A. Multirate margin and MTOP}

To reduce the MAC-layer overhead in multirate, multihop networks, this paper proposes a novel frame forwarding mechanism, called Multihop Transmission Opportunity (MTOP), where a frame is forwarded over multiple hops (say, $0 \rightarrow 1$, $1 \rightarrow 2,2 \rightarrow 3$, etc.) with a single channel contention as shown in Fig. 1(c). It extends the idea of TXOP but appears 


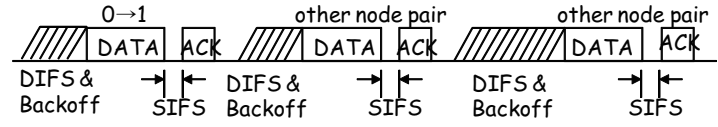

(a) 802.11

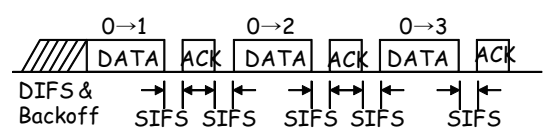

(b) TXOP

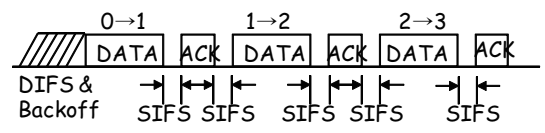

(c) MTOP

Fig. 1. Communication sequence in 802.11, TXOP and MTOP. (In (a), the MAC overhead such as DIFS and backoff is as high as $95 \%$ of total communication time, which is minimized in (b) and (c). In (b), node 0 sends three back-to-back frames. In (c), nodes 0,1 , and 2 relay a frame back-toback.)

more preferable in multihop networks. However, it could cause additional collisions because intermediate nodes ( 1 and 2) forward frames without appropriately contending for the medium.

While Section III of this paper is devoted to demonstrate that MTOP does not cause additional collisions in reality, this subsection briefly overviews it using the concept of multirate margin. Note that 802.11 MAC avoids collisions based on the defer-if-hear-anything principle or CSMA such that a node defers its transmission if it hears any signal stronger than a certain threshold, called defer threshold. It is typically set in a conservative manner to avoid any potential collisions. In multirate radios, it is tuned for the lowest-rate transmission (1Mbps in $802.11 \mathrm{~b}$ ), which becomes even more conservative or wasteful for high-rate transmissions.

This paper calls the difference between the pre-specified defer threshold (for the lowest-rate transmission) and the required defer threshold for high-rate transmission as multirate margin. According to our analysis in Section III, it is about 8.9 $\mathrm{dB}$ for transmission at $11 \mathrm{Mbps}(802.11 \mathrm{~b})$, which is equivalent to $271 \mathrm{~m}$ under the assumptions detailed in Section III. A small-scale experiment based on Universal Software Radio Peripheral (USRP) [12] and GNU Radio [13] demonstrates that it is about $5 \mathrm{~dB}$ between low-rate DBPSK (300kbps) and high-rate DQPSK (600kbps) modulation scheme as detailed in Section V-A. Multihop forwarding in MTOP does not cause additional collisions due to this margin.

We perform an extensive simulation study based on $n s-2$ [14] to evaluate the proposed MTOP protocol. As underlying multirate mechanism and multihop routing algorithm, we use Path-centric Rate Adaptation for Mobile Networks (PRAM) [15] and Ad-hoc On-demand Distance Vector (AODV) [16], respectively. PRAM is a simple multirate adaptation algorithm, where a source determines the data rate of a routing path for a particular destination and lets all links of the path use the same rate [15]. To know the baseline performance, we also simulated single-rate cases, where the single data rate is used for all control and data frames. It will be explained in more detail in Section V-B. Our evaluation study has indicated that PRAM (without MTOP) achieves better performance than single-rate cases but MTOP (with PRAM) outperforms. Compared to PRAM, MTOP improves the packet delivery ratio by as much as $12 \%$ and the average end-to-end packet delay is reduced by $12 \sim 43 \%$.

\section{B. Organization}

The rest of the paper is organized as follows. Section II overviews the characteristics of multirate radio and discusses performance anomaly in multirate networks. Section III analyzes the defer threshold at different data rates, and quantitatively provides the multirate margin via analysis. Section IV describes the proposed protocol, MTOP, which is followed by GNU Radio/USRP-based experiment and ns-2-based evaluation in Section V. Section VI overviews the previous multirate adaptation algorithms in WLANs and multihop networks. The paper concludes with Section VII.

\section{BACKGROUND}

\section{A. Multirate support in IEEE 802.11 standards}

According to IEEE 802.11 PHY-layer specifications [1], it supports 2.4 GHz Direct Sequence Spread Spectrum (DSSS) at the data rate of 1 and 2 Mbps using Differential Binary Phase Shift Keying (DBPSK) and Differential Quadrature Phase Shift Keying (DQPSK), respectively. A later standard, IEEE $802.11 \mathrm{~b}$, supports the same $2.4 \mathrm{GHz}$ DSSS at the additional data rate of 5.5 and 11 Mbps (Complementary Code Keying or CCK) that trade off interference tolerance for performance.

However, with the multirate capability, how does a receiver know the data rate that is used by the transmitter? This is achieved by using two different data rates when transmitting a frame at the PHY layer, which is called Physical Layer Convergence Procedure (PLCP) frame. The PLCP frame is composed of PLCP preamble, PLCP header and the payload. In 802.11, the PLCP preamble and the PLCP header shall be transmitted using the lowest data rate $(1 \mathrm{Mbps})$. Meanwhile, the payload is transmitted at a higher rate, which is specified in the PLCP header.

\section{B. PHY- and MAC-layer overheads}

To analyze the PHY-layer overhead, let us consider a 512byte payload. Since the PLCP preamble and header are 144bits and 48bits $(192 \mu \mathrm{s})$, the overall frame size is $4288 \mu$ s at $1 \mathrm{Mbps}$ $\left(t_{1}\right)$. Since the payload can be transmitted at higher rates, it becomes $2240 \mu \mathrm{s}\left(t_{2}\right), 937 \mu \mathrm{s}\left(t_{5.5}\right)$, and $564 \mu \mathrm{s}\left(t_{11}\right)$ for 2 , 5.5 and $11 \mathrm{Mbps}$, respectively. Correspondingly, the per-frame PHY overhead due to PLCP preamble and header is $4.5 \%$, $8.6 \%, 20.5 \%$ and $34.0 \%$ at $1,2,5.5$ and $11 \mathrm{Mbps}$.

To analyze the MAC-layer overhead, note the frame sequence in 802.11 MAC. We assume no RTS/CTS exchange and assume a high traffic condition in which every frame transmission contend for medium access by waiting a random time chosen within the contention window $(C W) . C W$ is 
$31 \sim 1023$ and slot time is $20 \mu \mathrm{s}$. The time for contention on the average is $16 \times 20$ or $320 \mu$ s when $C W$ is 31 .

Now, the time duration for the frame sequence at data rate $i, T_{i}$, consists of DIFS and contention $\left(t_{c}\right.$ or $\left.50+320 \mu \mathrm{s}\right)$, Data $\left(t_{i}\right)$, SIFS $\left(t_{S I F S}\right.$ or $\left.10 \mu \mathrm{s}\right)$ and $\mathrm{ACK}\left(t_{A C K}\right.$ or $\left.376 \mu \mathrm{s}\right)$, i.e.,

$$
T_{i}=t_{c}+t_{i}+t_{S I F S}+t_{A C K} .
$$

It totals $5044 \mu \mathrm{s}\left(T_{1}\right), 2996 \mu \mathrm{s}\left(T_{2}\right), 1693 \mu \mathrm{s}\left(T_{5.5}\right)$, and $1320 \mu \mathrm{s}$ $\left(T_{11}\right)$ for $1,2,5.5$ and $11 \mathrm{Mbps}$, respectively. Considering the payload size, the MAC-layer overhead amounts to $15.0 \%$, $25.2 \%, 44.7 \%$ and $57.3 \%$ for $1,2,5.5$ and $11 \mathrm{Mbps}$, respectively. In other words, $57.3 \%$ of channel time is wasted due to the MAC overhead at $11 \mathrm{Mbps}$, which is increased to as much as $95.0 \%$ when $C W$ is 1023 . It is evident, therefore, that reducing this overhead is critically important, particularly for high-rate transmissions.

Efforts have been made to reduce the PHY and MAC overheads. For example, a later standard $802.11 \mathrm{~b}$ introduces a shorter PLCP preamble (72bits) and allows the PLCP header to transmit at $2 \mathrm{Mbps}$ for high-rate transmission (5.5 and $11 \mathrm{Mbps}$ ), reducing per-frame PHY overhead from $192 \mu$ s to $96 \mu \mathrm{s}$. Also, 802.11a and 802.11g reduce the MAC overhead by adopting a smaller $C W(15 \sim 1023)$ as well as a smaller slot size $(9 \mu \mathrm{s})$.

\section{Transmission opportunity and performance anomaly}

Another important development in reducing the MAC overhead is Transmission Opportunity (TXOP), which allows a node to transmit multiple frames with a single channel access. This was originally proposed in 802.11 e to improve fairness by granting a node with lower channel access priority a dedicated time duration, which is called $T X O P_{\text {limit }}$ (3264 or $6016 \mu \mathrm{s}$ ) [8].

In fact, the fairness problem and the associated performance anomaly have been observed by many researchers in the context of multirate WLANs [10], [11], [17]. While 802.11 MAC guarantees that each node gets an equal chance of transmitting its frames, it does not necessarily mean that each node gets an equal share of the channel (time) in a multi-rate environment. With TXOP, low-rate node pair is not impacted significantly in terms of throughput but high-rate node pair is benefited significantly.

To demonstrate this, consider an example scenario in Table I(a) for a mixture of low $(A \leftarrow B)$ and high-rate $(C \rightarrow D)$ communication. $B$ transmits one 512-byte frame during $T_{1}$ or $5044 \mu \mathrm{s}$ and $C$ does one during $T_{11}$ or $1320 \mu$ s. Assuming that the two transmitters get equal chance of medium access, the aggregate throughput is

$$
\frac{\text { Two } 512 B \text { frames }}{T_{1}+T_{11}}=1.29 \mathrm{Mbps},
$$

which is barely larger than the lower bit rate. Moreover, $C$ - $D$ node pair is not fairly treated because it uses only $21 \%$ of medium time $\left(\frac{T_{11}}{T_{1}+T_{11}}\right)$.

Before discussing how TXOP improves the situation, let us compute the maximum allowable number of frames $\left(k_{i}\right)$ to
TABLE I

Performance anomaly. (In (A), A-B: 1Mbis, 272M, C-D: 11Mbis, 118M. IN (B), A-B: 1MBPS, 272M, C-TO-D: 11MBPS, 118M EACH HOP.)

(a) Single-hop scenario

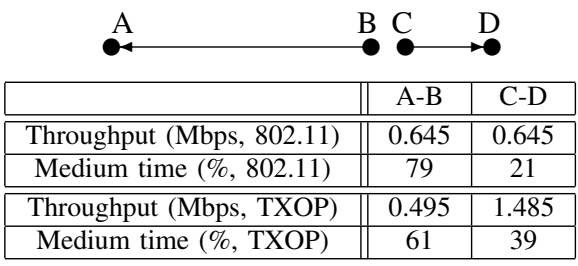

(b) Multihop scenario

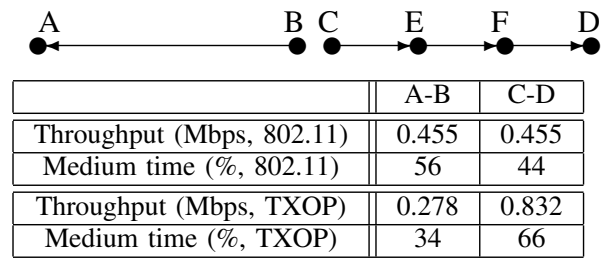

transmit consecutively at data rate $i$ during $T X O P_{\text {limit }}$. Since SIFS $\left(t_{S I F S}\right)$ replaces DIFS and contention $\left(t_{c}\right)$ between $k_{i}$ consecutive frames, $k_{i}$ can be obtained as follows:

$\max _{k_{i}}\left(k_{i} \cdot T_{i}-\left(k_{i}-1\right) \cdot t_{c}+\left(k_{i}-1\right) \cdot t_{S I F S} \leq T X O P_{\text {limit }}\right)$.

When $T X O P_{\text {limit }}$ is $3264 \mu \mathrm{s}$ [8], $k_{i}$ is 1 for 1 and $2 \mathrm{Mbps}, 2$ for $5.5 \mathrm{Mbps}$ and 3 for $11 \mathrm{Mbps}$. Now, while node $B$ transmits one frame $\left(k_{1}\right)$ during $T_{1}$, node $C$ transmits three frames $\left(k_{11}\right)$ consecutively during $T_{11}^{\prime}=3 \cdot T_{11}-2 \cdot t_{c}+2 \cdot t_{S I F S}$ or $3240 \mu \mathrm{s}$. Therefore, the aggregate throughput is improved to

$$
\frac{\text { Four } 512 B \text { frames }}{T_{1}+T_{11}^{\prime}}=1.98 \text { Mbps. }
$$

More importantly, $C$ - $D$ node pair uses $39 \%$ of medium time, which is a significant improvement in terms of fairness. Please refer to Table I(a) for summary.

\section{Multihop anomaly}

In a multihop network, the problem becomes more complicated due partly to inter-hop interference and rate-hop count tradeoff. Consider an example in Table $\mathrm{I}(\mathrm{b})$, where $B$ wants to talk to $A$ at $1 \mathrm{Mbps}$ and $C$ wants to talk to $D$ at $11 \mathrm{Mbps}$ with two intermediate nodes, $E$ and $F$. Note that the communication range at 1 and $11 \mathrm{Mbps}$ is $272 \mathrm{~m}$ and $118 \mathrm{~m}$, respectively, as detailed later in this paper. Two transmitters $(B$ and $C$ ) and two intermediate nodes $(E$ and $F$ ) will get an equal chance for medium access. With 802.11, the aggregate end-to-end throughput will be

$$
\frac{\text { Two } 512 B \text { frames }}{T_{1}+3 \cdot T_{11}}=0.91 \mathrm{Mbps},
$$

and $C$ - $D$ node pair occupies $\frac{3 \cdot T_{11}}{T_{1}+3 \cdot T_{11}}$ or $44 \%$ of medium time, which indicates no serious fairness problem (less channel time per hop but more hops end-to-end).

Interestingly, TXOP deteriorates fairness in multihop networks. Since $C, E$ and $F$ will transmit three frames at once 

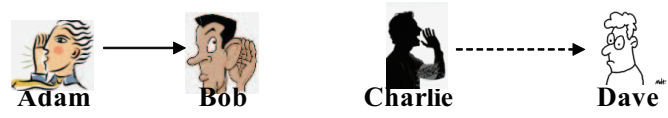

(a) Charlie does not talk because it would bother Bob
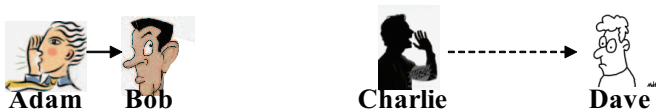

(b) Charlie talks because it does not bother Bob
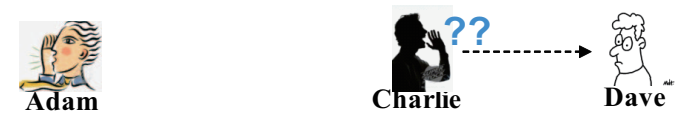

(c) Considering the worst case, Charlie does not talk
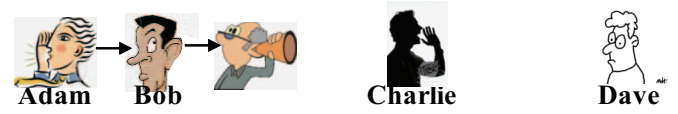

(d) Knowing Charlie does not talk, Adam \& Bob exploit it

Fig. 2. The defer-if-hear-anything principle. (The same, low defer threshold is employed resulting in the multirate margin as in (c). Multihop forwarding is drawn in (d).)

during $T_{11}^{\prime}$ each, the aggregate end-to-end throughput will be

$$
\frac{\text { Four } 512 B \text { frames }}{T_{1}+3 \cdot T_{11}^{\prime}}=1.11 \mathrm{Mbps},
$$

and $C$ - $D$ node pair occupies $\frac{3 \cdot T_{11}^{\prime}}{T_{1}+3 \cdot T_{11}^{\prime}}$ or $66 \%$ of medium time. In other words, TXOP increases the aggregate throughput slightly but degrades fairness as the priority is given to highrate communications. More seriously, $A-B$ pair achieves only about a half of the throughput in comparison to 802.11 in Table I(b). Unlike in single-hop networks, TXOP greatly impacts the low-rate communication. In practice, TXOP would perform even worse because opportunities are not always utilized.

\section{Multirate MARGin}

\section{A. An Illustrative Example}

Another possibility to reduce the MAC overhead is to extend the idea of TXOP in multihop environment. This subsection illustrates an example that explains multirate margin and multihop forwarding mechanism in the proposed MTOP protocol. Consider the voice communication among four persons as in Fig. 2: Adam, Bob, Charlie and Dave. Adam wants to talk to Bob and Charlie wants to talk to Dave. They use the same defer-if-hear-anything principle (like CSMA) and non-negligible inter-message pause (like DIFS and backoff) to avoid collisions.

In Fig. 2(a), when Adam talks to Bob, Charlie would not begin his conversation to Dave because he knows it would interfere Adam-Bob's communication (analogous to low defer threshold at 1Mbps). In Fig. 2(b), Adam-Bob's conversation would not be interfered by Charlie's talk because Bob sits close to Adam, and so Charlie begins to talk to Dave concurrently with Adam (high defer threshold at 11Mbps). In reality, however, Charlie does not know whom Adam talks to but just overhears Adam as shown in Fig. 2(c). Considering the worst-case scenario, Charlie would not begin his conversation
TABLE II

CHARACTERISTICS OF AN 802.11B MULTI-RATE RADIO. (TRANSMIT POWER: 15 DBM, INDOOR RADIO PROPAGATION MODEL WITH PATH LOSS EXPONENT OF 3.3 [18].)

\begin{tabular}{|c||c|c|c|c|}
\hline Data rate $(\mathrm{Mbps})$ & 1 & 2 & 5.5 & 11 \\
\hline \hline Receive sensitivity $(\mathrm{dBm})$ & -94 & -91 & -87 & -82 \\
\hline Range or $r_{i}(\mathrm{~m})$ & 272 & 221 & 167 & 118 \\
\hline \hline SIR requirement $(\mathrm{dB})$ & 2.2 & 5.2 & 4.4 & 7.6 \\
\hline Max. interference $(\mathrm{dBm})$ & -96.2 & -96.2 & -91.4 & -89.6 \\
\hline Min. RI distance $(\mathrm{m})$ & 317 & 317 & 227 & 200 \\
\hline Min. TI distance $(\mathrm{m})$ & 589 & 538 & 394 & 318 \\
\hline Defer threshold $(\mathrm{dBm})$ & -105.1 & -103.8 & -99.3 & -96.2 \\
\hline
\end{tabular}

$*$ Values in the last five rows are for target BER of $10^{-5}$. SIR requirement is explained in Section III-B. Other four are explained in Section III-C.

until Adam completes (a lower one is specified as the defer threshold). In Fig. 2(d), knowing that Charlie would not talk, Bob exploits this opportunity to immediately forward the message to the next person. Time is saved because Bob does not "pause" between the messages.

\section{B. Receive sensitivity and SINR requirement}

Steps to analyze the multirate margin are as follow: (i) Estimate the communication range $\left(r_{i}\right)$ based on the receive sensitivity at different rates. (ii) Estimate the SIR requirement using analysis. (iii) Receive sensitivity is subtracted from the SIR requirement for target BER of $10^{-5}$ to estimate the maximum tolerable interference, which translates to the minimum RI (receiver to interference) distance. (iv) This is added to the communication range to estimate the minimum TI (transmitter to interferer) distance, which is translated to the required defer threshold at different rates based on the transmit power and path loss model. (v) Finally, multirate margin is the difference between the defer threshold at $1 \mathrm{Mbps}$ and the required defer threshold at high rates. Table II summarizes the results.

Step (i): For a successful communication, the received signal power must be higher than the receive sensitivity in the presence of path loss over distance. Table II shows them at four data rates of $2.4 \mathrm{GHz} 802.11 \mathrm{~b}$ radio [19]. Indoor path loss model by Marquesse [20] has been used to derive the communication range, i.e. path loss $=40.2+20 \cdot \log _{10}(d)$ if $d \leq 8 m$, and $58.5+33 \cdot \log _{10}(d / 8)$, otherwise.

Step (ii): Moreover, the received signal power must be strong enough to overcome the influence of noise and interference from all other simultaneous transmissions, i.e., SINR must be higher than a certain threshold [21]. A higher-rate communication requires a higher threshold, which means that it is more subjective to interference. Based on the study in [18], BER calculation for $802.11 \mathrm{~b} 1 \mathrm{Mbps}$ is as follows:

$$
B E R_{1}=Q(\sqrt{11 \cdot S I R})
$$

where $Q$ function is defined as

$$
Q(x)=\frac{1}{\sqrt{2 \pi}} \int_{x}^{\infty} e^{-\left(\frac{t^{2}}{2}\right)} d t .
$$

BER calculation for $802.11 \mathrm{~b} 2,5.5$ and $11 \mathrm{Mbps}$ are given as follows:

$$
B E R_{2}=Q(\sqrt{5.5 \cdot S I R})
$$




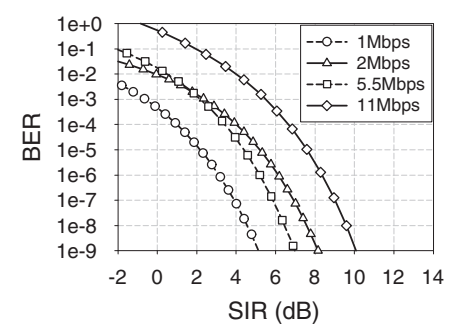

(a) BER versus SIR

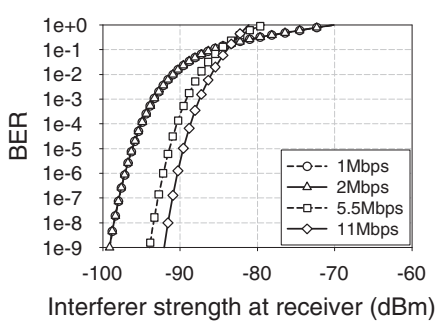

(b) BER versus interferer strength at the receiver

Fig. 3. Multirate margin. (Fig. (b) shows the highest tolerable interference when the signal strength at the receiver is equal to the receive sensitivity.)

$$
B E R_{5.5} \leq \frac{2^{4-1}}{2^{4}-1}(14 \cdot Q(\sqrt{8 \cdot S I R})+Q(\sqrt{16 \cdot S I R}))
$$

and

$$
\begin{array}{r}
B E R_{11} \leq \frac{2^{8-1}}{2^{8}-1}(24 \cdot Q(\sqrt{4 \cdot S I R})+16 \cdot Q(\sqrt{6 \cdot S I R})+ \\
174 \cdot Q(\sqrt{8 \cdot S I R})+16 \cdot Q(\sqrt{10 \cdot S I R})+ \\
24 \cdot Q(\sqrt{12 \cdot S I R})+Q(\sqrt{16 \cdot S I R})) .
\end{array}
$$

Fig. 3(a) shows the BER curve for four different data rates. The SIR requirement for target BER of $10^{-5}$ is shown in Table II. Note that we use SIR instead of SINR as in [18] because interference is generally much stronger than noise [22] and the capacity of multihop networks is determined by the communication robustness in the presence of co-channel interference.

\section{Defer threshold}

Step (iii): Assume that the signal strength at the receiver is equal to the receive sensitivity in Table II, Fig. 3(b) shows the maximum tolerable interference to meet the SIR requirement of Fig. 3(a). Those for the target BER of $10^{-5}$ are shown in Table II along with the equivalent RI distance. For instance, nodes within $317 \mathrm{~m}$ from an 1 Mbps "receiver" must not transmit concurrently; otherwise, the communication will fail due to the lower SIR than required.

Step (iv): In order to refrain a potential interferer from transmitting, defer threshold is employed. In other words, an 802.11 PHY performs Clear Channel Assessment (CCA), which involves declaring the channel busy if it detects any signal energy above the pre-specified defer threshold [23].

However, since the receiver does not transmit signals, it can only be mandated in practice by sensing the signal from the transmitter. Therefore, nodes within 589m from an $1 \mathrm{Mbps}$ "transmitter" must not transmit concurrently, which is obtained by adding the communication range $\left(r_{1}\right)$ to the RI distance [24]. This minimum TI distance is translated to the defer threshold by using the indoor path loss model and the transmit power of $15 \mathrm{dBm}$.

\section{Multirate margin}

As discussed above, transmissions at different rates require different defer thresholds but, in practice, it is unavoidable to

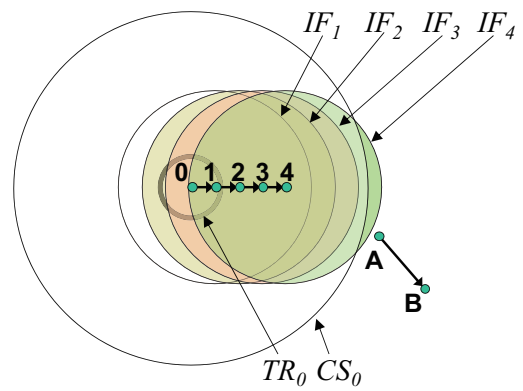

(a) $11 \mathrm{Mbps}$

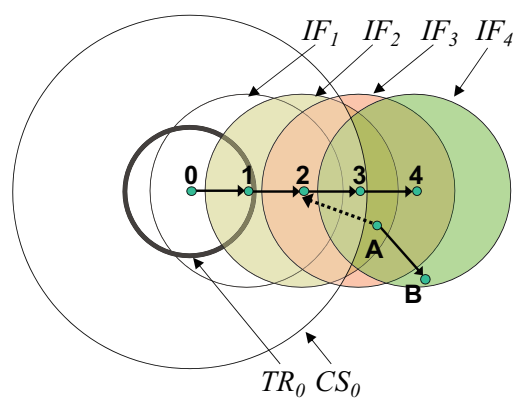

(b) $1 \mathrm{Mbps}$

Fig. 4. The MTOP mechanism. ( $A \notin I F_{2}$ in (a) but $A \in I F_{2}$ in (b). Therefore, nonstop forwarding $1 \rightarrow 2$ will be successful in (a) but not in (b).)

employ the same, lowest threshold [25], which is $-105.1 \mathrm{dBm}$ as shown in Table II (see Fig. 2(c) for analogy).

Now, since the required defer threshold at $11 \mathrm{Mbps}$ is -96.2 $\mathrm{dBm}$, there exists an $8.9 \mathrm{~dB}$ margin, which leaves a room for improvement. We call it multirate margin in this paper. The MTOP protocol exploits this margin by allowing a frame to travel 1-2 more hops with a single medium access, which we will discuss in more detail in the next section.

\section{Multihop Transmission Opportunity (MTOP)}

This section presents Multihop Transmission OPportunity (MTOP). Three key components of MTOP are: multihop forwarding (section IV-A), combination of MTOP with TXOP (section IV-B) and PRAM routing algorithm (section IV-C).

\section{A. Multihop forwarding}

Multihop forwarding in MTOP extends the idea of TXOP over multiple consecutive hops in multirate multihop networks as shown in Fig. 4(a). In the figure, $C S_{0}$ and $I F_{1}$ denote the area defined by the minimum TI distance of transmitter 0 and by the minimum RI range of receiver 1, respectively. (CS and IF range stand for carrier sense and interference range, respectively.) While node 0 transmits a frame to node 1 , node $A$ is allowed to transmit its own frame to node $B$ because $A$ is outside of $C S_{0}$, or $A \notin C S_{0}$. When node 1 forwards the frame to node 2 based on the MTOP mechanism, it would not be subject to interference because $A \notin I F_{2}$. This holds true for the following hop node as well because $A \notin I F_{3}$. In other words, when node 0 transmits data frame at $11 \mathrm{Mbps}$, most of potential interferers for the current communication 
$(0 \rightarrow 1)$ as well as the next two hop communications $(1 \rightarrow 2$ and $2 \rightarrow 3$ ) would be inhibited. This is due to the additional 8.9dB margin discussed earlier in Section III-D. On the other hand, this scenario changes at low-rate communication. As shown in Fig. 4(b), node 1's forwarding to node 2 will be interfered by $A$ 's transmission because $A \in I F_{2}$.

We define $M T O P_{\text {limit }}$ as the remaining margin that a node can exploit for successive transmission to the next hop without an additional contention for medium access. While $T X O P_{\text {limit }}$ is measured in time and is associated with a node, $M T O P_{\text {limit }}$ is measured in $\mathrm{dB}$ and is associated with a frame. However, similar to $T X O P_{\text {limit }}, M T O P_{\text {limit }}$ can be translated to the number of hops, for convenience. It is not difficult to estimate the maximum allowable number of hops $\left(h_{i}\right)$ to forward at data rate $i$ as

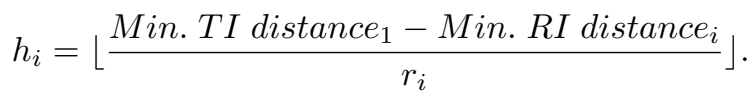

According to parameters in Table II, $h_{i}$ is one for $1 \mathrm{Mbps}$ and $2 \mathrm{Mbps}$, two for $5.5 \mathrm{Mbps}$, and three for $11 \mathrm{Mbps}$.

\section{B. MTOP and TXOP}

It is noted that TXOP and MTOP are not mutually exclusive and can be combined to diversify and maximize the transmission opportunities in multirate multihop networks. When a node transmits a frame, it makes a prudent decision whether to seek an additional transmission opportunity according to TXOP or MTOP. For example, a node transmits a frame at $5.5 \mathrm{Mbps}$ and the next frame to transmit is a $2 \mathrm{Mbps}$ frame. No additional opportunity is available via TXOP because it will exceed $T X O P_{\text {limit }}$. Similarly, no opportunity is utilized via TXOP when there is no more frames to transmit in the queue, which is likely to happen very often. In both cases, the node seeks an opportunity via MTOP.

\section{Multi-rate routing algorithm}

For MTOP to be useful, a rate-aware routing algorithm is needed. Proactive routing algorithms, such as DSDV [26], have been used in most of previous studies [3], [25], [27], [28]. They could be a reasonable choice in static, multirate networks, but incur a high control overhead in mobile networks because a larger volume of route information must be exchanged more frequently. In other words, each node is required to keep track of and exchange the status of all its links at all available data rates increasing the control overhead. We chose an on-demand routing protocol, AODV [16], as the underlying network-layer protocol.

In conjunction with AODV, a multirate adaptation algorithm, PRAM [15], is used in this paper. It finds an optimal data rate for a routing path and adapts it dynamically based on path lifetime. In other words, a source node floods a RREQ (route request control packet) to find a routing path as in the original AODV. Unique to PRAM is to dictate a data rate for RREQ and to enforce every intermediate node to use the same rate when forwarding RREQ. It discovers suboptimal routing paths at the benefit of simplicity and lower control overhead. Please refer to [15] for details.

\section{Performance Evaluation}

\section{A. Multirate margin via USRP/GNU Radio-based experimen-} tation

Since radio propagation and its channel dynamics cannot easily be captured using analytical or simulation models, we conducted an experimental study to demonstrate the multirate margin based on a small-scale testbed using USRP [12] and GNU Radio [13].

The following are the details of the experiment (see [29] for a similar setup). (i) The testbed includes 3 USRP systems (version 5b), 3 RFX2400 transceivers (2.3-2.9 GHz) and GNU Radio software (version 3.1.3). (ii) Modulation schemes used are DBPSK (low-rate) and DQPSK (high-rate). (iii) Carrier frequency and bandwidth we have tested are $2.4835 \mathrm{GHz}$ and $300 \mathrm{KHz}$, respectively. Therefore, the maximum data rate is $300 \mathrm{Kbps}$ and $600 \mathrm{Kbps}$ for DBPSK and DQPSK, respectively. A smaller bandwidth and data rates are used partly due to bandwidth constraints imposed by the USRP [30]. (iv) Transmitter amplitude is set to 8,000 , which is smaller than the default value $(12,000)$. This is to make the communication range no farther than 300 feet $^{1}$. (v) Packet size is 1,500 bytes and 3,300 packets were transmitted for each experiment.

Our goal is to observe a similar trend as in Table II, particularly the multirate margin with two data rates supported by DBPSK and DQPSK modulation schemes. The experiment has been conducted in two phases. First, in order to obtain communication range $\left(r_{i}\right)$ with DBPSK and DQPSK, we set up two USRP systems and measured received signal strength indicator (RSSI) versus distance and packet delivery ratio (PDR) versus $\mathrm{RSSI}^{2}$. According to our experimental results in Figs. 5(a) and 5(b), $r_{i}$ for DBPSK and DQPSK is estimated as $215 \mathrm{ft}$ and $150 \mathrm{ft}$, respectively. Note that $90 \%$ PDR is used to estimate the communication range, which is equivalent to BER of $10^{-5}$.

Second, in order to obtain the minimum RI distance, we set up three USRPs, a transmitter $(T)$, a receiver $(R)$ and an interferer $(I)$ on a straight line $(T-R-I)$. The TR distance is fixed to the communication range, i.e., $215 \mathrm{ft}$ and $150 \mathrm{ft}$ for DBPSK and DQPSK, respectively. Figs. 5(c) and 5(d) show BER versus SIR and BER versus RSSI (from $I$ to $R$ ), which must be compared to Figs. 3(a) and 3(b), respectively. Note that SIR at the receiver is calculated as RSSI from the sender minus RSSI from the interferer [32].

According to the experiment results, we observed that the low-rate communication (DBPSK) is more robust to interference than high-rate (DQPSK) as similarly observed in [30]. Minimum RI distance is estimated as $220 \mathrm{ft}$ and $235 \mathrm{ft}$ for DBPSK and DQPSK, respectively, and the minimum TI distance for $90 \%$ PDR is about $435 \mathrm{ft}$ and $385 \mathrm{ft}$. Comparing to Table II, we can conclude that the same trend and the multirate margin at a high rate (435 versus $385 \mathrm{ft}$ ) has been observed.

\footnotetext{
${ }^{1}$ This experiment was conducted in the Edgewater Park near Lake Erie in Cleveland, $\mathrm{OH}$.

${ }^{2}$ Note also that RSSI obtainable from USRP/GNU Radio is "digital RSSI" value, meaning that it is based on the output of the analog-to-digital converter (ADC), which is not the true RF power at the antenna [31].
} 


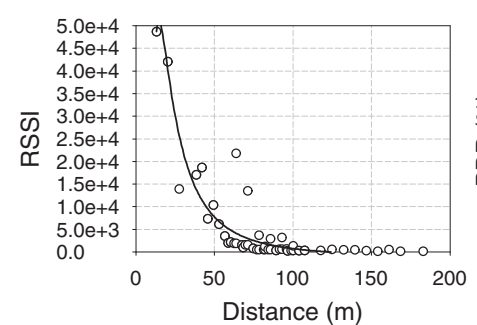

(a) RSSI versus distance

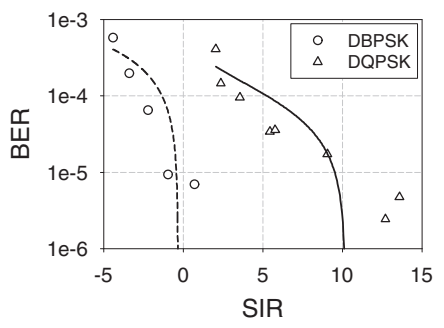

(c) BER versus SIR

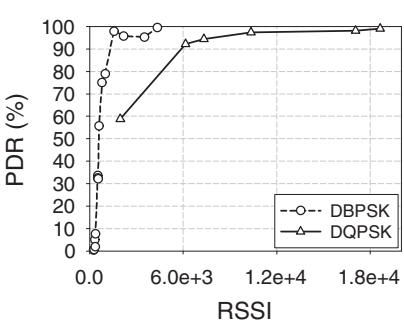

(b) PDR versus RSSI

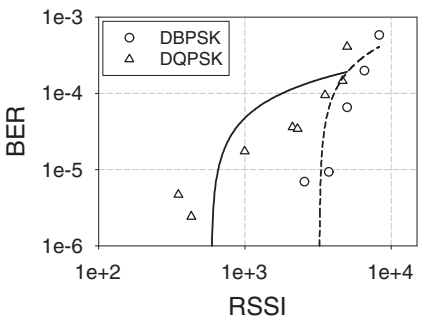

(d) BER versus interference strength (RSSI) at the receiver
Fig. 5. USRP/GNU Radio-based experimental results.

\section{B. Simulation environment}

It is generally understood that the implementation of CSMA is hard for the current USRP/GNU Radio platform due to hardware limitations [33]-[35]. Instead, the performance of MTOP is evaluated via ns-2 [14], which simulates node mobility, a realistic physical layer, radio network interfaces, and the 802.11 MAC protocol. We used 802.11a radios instead of $802.11 \mathrm{~b}$ because there are more data-rate choices in $802.11 \mathrm{a}$ $(6,9,12,18,24,36,48$, and 54Mbps). Table III shows four out of eight bit rates in 802.11a.

Our evaluation is based on the simulation of $20 \sim 140$ mobile nodes located in an area of $1500 \times 300 \mathrm{~m}^{2}$. The data traffic simulated is constant bit rate (CBR) traffic. 20 CBR sessions are simulated at the rate of 20 packets/second. The random waypoint mobility model [36] is used in our experiments with the minimum and maximum node speed of $1 \mathrm{~m} / \mathrm{s}$ and $5 \mathrm{~m} / \mathrm{s}$, and a pause time of 0 second. With this mobility model, a node travels (between 1 and $5 \mathrm{~m} / \mathrm{s}$ ) towards a randomlyselected destination in the network. After the node arrives at the destination, it travels towards another randomly selected destination. Simulation time is 300 seconds for each run.

The aforementioned simulation parameters are typical in many previous studies on mobile ad hoc networks including [37] except that the traffic intensity and the number of nodes $(N)$ are higher than normal. The traffic intensity of 20 sessions with 20 packets/s each could be overwhelming at $6 \mathrm{Mbps}$ but it can be reasonably handled at $54 \mathrm{Mbps} . N$ is as many as 140 in our simulation study because we're more interested in high-density scenarios.

We compare 11 different schemes: fixed data rate of 6 $\sim 54 \mathrm{Mbps}$ (8 cases), PRAM, TXOP (+PRAM), and MTOP (+PRAM). In fixed rate cases, every data packet is transmitted at the specified data rate. In a sparse network (e.g., 20 nodes in
TABLE III

CHARACTERISTICS OF AN 802.11A MULTI-RATE RADIO AND $M T O P_{\text {limit }}$ $\left(h_{i}\right)$. (TRANSMIT POWER IS 6 DBM AND TWO-RAY GROUND RADIO PROPAGATION MODEL WITH PATH LOSS EXPONENT OF 4.0 [14] IS USED. FOUR OUT OF EIGHT DATA RATES ARE SHOWN FOR BREVITY.)

\begin{tabular}{|c||c|c|c|c|}
\hline Data rate $(\mathrm{Mbps})$ & 6 & 18 & 36 & 54 \\
\hline \hline Receive sensitivity $(\mathrm{dBm})$ & -82 & -77 & -70 & -65 \\
\hline Range $(\mathrm{m})$ & 238 & 178 & 119 & 89 \\
\hline SIR requirement $(\mathrm{dB})$ & 6.02 & 10.79 & 18.80 & 24.56 \\
\hline$M T O P_{\text {limit }}\left(h_{i}\right)$ & 1 & 2 & 3 & 4 \\
\hline
\end{tabular}

the network), we expect the 54 Mbps case suffers most because of the connectivity problem. But it will become advantageous as $N$ increases. Performance metrics are packet delivery ratio (PDR) and average packet delay. Since MTOP encourages faster, nonstop frame forwarding and thus potentially causes additional collisions, we also measured the number of frame drops at the MAC layer. For MTOP, statistics on the mixture of data rates used and the corresponding utilization of frame forwarding opportunity are also presented to understand the adaptive behavior of MTOP.

\section{Simulation results}

Fig. 6 compares PDR and average packet delay of 6 54Mbps, PRAM, TXOP, and MTOP. (For readability, only two cases of fixed rate, $6 \mathrm{Mbps}$ and $54 \mathrm{Mbps}$, are shown in the figure.) Fig. 6(a) shows the PDR versus $N$. The 54Mbps case does not function well as shown in the figure, particularly with a small $N$. This is due mainly to the lack of end-to-end connectivity. However, its performance increases rapidly as $N$ increases. In the 6Mbps case, the PDR is the highest when $N$ is 20 and decreases as $N$ increases due to the increased control traffic (broadcast of RREQs) as $N$ increases.

PRAM and TXOP in general achieve a better performance than $6 \mathrm{Mbps}$ and $54 \mathrm{Mbps}$ cases because they use a combination of all available data rates to maximize the network performance. However, as shown in Fig. 6(a), when $N$ is larger than 100, the $54 \mathrm{Mbps}$ case performs better than PRAM. It is not surprising that the fixed rate case performs better than PRAM as it does not cost any extra overhead to determine data rates. On the other hand, MTOP outperforms 6Mbps, $54 \mathrm{Mbps}$, PRAM, and TXOP in the entire range of $N$ simulated as shown in Fig. 6(a). Fig. 6(b) shows the average packet delay versus $N$. The $6 \mathrm{Mbps}$ case experiences the largest packet delay because of its slow packet transmission speed. The $54 \mathrm{Mbps}$ case shows the lowest packet delay in the entire range of $N$. However, it does not represent its true performance because its PDR is low too and the computation of the average packet delay does not take the lost packets into account. Comparing PRAM, TXOP and MTOP, they show a similar trend while MTOP exhibits the lowest packet delay.

As discussed earlier, MTOP may cause more collisions because an intermediate node is allowed to forward a frame when it receives it at high rates. Fig. 7(a) shows the number of packet drops versus $N$. PRAM drops more packets than MTOP as shown in the figure. In case of MTOP, there are two types of packet drops, those at the first-hop (non- 


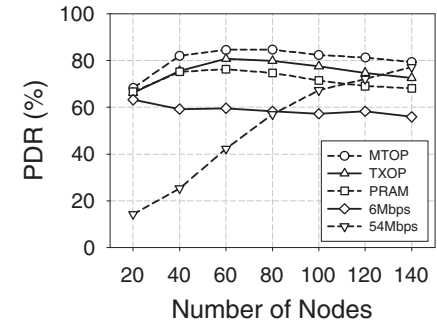

(a) PDR

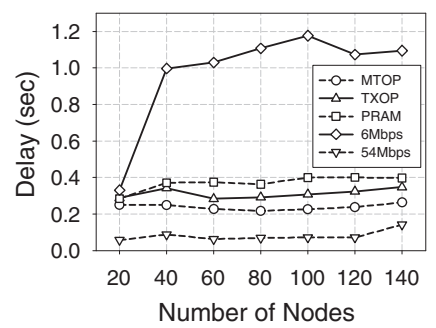

(b) Average packet delay (sec.)
Fig. 6. Performance of MTOP in comparison with fixed rate cases and PRAM

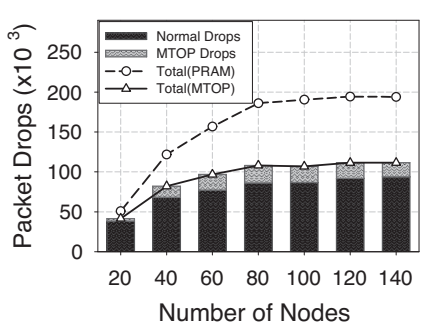

(a) Number of packet drops

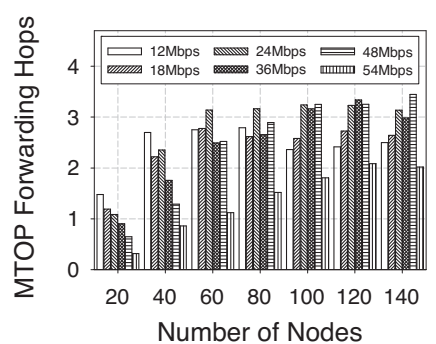

(b) MTOP forwarding hops (including the first-hop)
Fig. 7. Comparison of packet drops and the average number of MTOP forwarding

opportunistic) communication (normal drops) and others at the next-hop (opportunistic) forwarding via the MTOP mechanism (MTOP drops). As shown in the figure, MTOP drops are as high as $18 \%$ of normal drops, proving that MTOP's multihop forwarding does not cause ant serious collision problem.

Fig. 7(b) shows how many hops are traversed using the MTOP forwarding mechanism. For example, when $N=20$, communication at $12 \mathrm{Mbps}$ traverses 1.4 hops on the average. Excluding the first-hop communication, which is based on competition, MTOP additionally forwards a frame at $12 \mathrm{Mbps}$ 0.4 hops, on the average. The figure does not show $6 \mathrm{Mbps}$ and $9 \mathrm{Mbps}$ case because they does not use the MTOP forwarding. The general trend is that there are more forwarding when the network is denser, which is because there exists more neighbors to utilize as intermediate nodes. It is also observed that, when node density is low, low-rate communications have utilized the MTOP opportunity more, although they are given less chances. On the other hand, as node density increases, high-rate communications utilize more MTOP opportunities, forwarding frames to next hop nodes. MTOP adaptively adjust its behavior to optimize the network performance.

In order to understand how MTOP improves the network performance, we collect statistics about the data rate used in the networks. Figs. 8(a) and 8(b) show the mixture of data rates for 20- and 140-node networks, respectively. In the 20node network, for example, the mixture is $12 \%, 14 \%, 15 \%$, $23 \%, 14 \%, 9 \%, 1 \%$, and $12 \%$ for 6 54 Mbps. About $80 \%$ of routing paths use $6 \sim 24 \mathrm{Mbps}$. On the other hand, in the 140 -node network, the mixture becomes $2 \%, 1 \%, 3 \%, 10 \%$, $24 \%, 18 \%, 9 \%$, and $33 \%$ and around $95 \%$ of routing paths

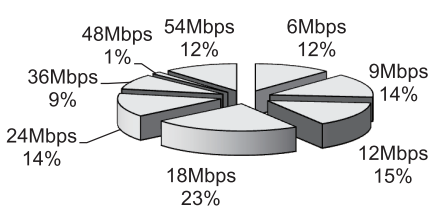

(a) Data rates used by source node (20-node network)

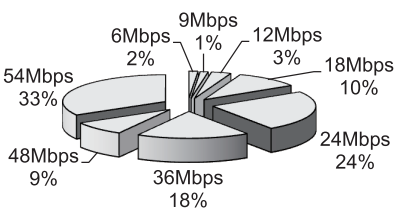

(b) Data rates used by source node (140-node network)
Fig. 8. Performance of MTOP. (In the 20-node network, low rate communications dominate the network as shown in (a). More nodes in the 140-node network have a chance to use high data rates as in (b).)

use 18 54 Mbps. In other words, MTOP uses more low-rate transmissions when network is sparse and uses more high-rate transmissions when network is dense.

\section{RELATED WORK}

To exploit multirate capability in WLANs, there have been a number of proposals on multirate algorithms for 802.11based WLANs in the literature. They can be categorized as sender- and receiver-based. Auto-Rate Fallback (ARF) [38] is the first multirate algorithm, which is sender-based and was designed to optimize the application throughput in WaveLANII devices. The basic idea of ARF is to use a higher rate upon consecutive successful transmissions and to fall back to a lower rate after a number of consecutive transmission failures. Receiver-Based Auto Rate (RBAR) [27] is a receiver-based multirate algorithm. It assumes to use the RTS/CTS handshaking. Upon receiving an RTS frame, the receiver estimates the channel quality based on the SINR of the received RTS frame and then determines the best data rate that the transmitter must use. The estimated optimal rate is then sent back to the sender piggybacking in the CTS packet. Opportunistic Auto Rate (OAR) protocol [9] is built on a multirate algorithm such as ARF or RBAR and improves the network performance by exploiting durations of high-quality channel conditions and sending multiple back-to-back data packets.

A great deal of work on multi-rate adaptation has been reported in the context of multihop networks [3], [25], [27], [28] because it can greatly improve the network throughput. They can be categorized as proactive or on-demand depending on the routing algorithm used. With a proactive multi-rate algorithm, each node maintains link costs to each of its neighbors while taking the multirate capability into account. Link costs used include bandwidth distance product (BDiP) [25], medium time metric (MTM) [39], estimated transmission time (ETT, MIT's Roofnet) [3], and weighted cumulative ETT (WCETT, MSR's testbed) [40]. Unlike those mentioned above, few works have been done in combination with ondemand routing algorithms such as DSR [36] and AODV [16]. SampleRate [3] and Robust Rate Adaptation Algorithm (RRAA) [41]) are designed to work with on-demand routing algorithms but they target static networks.

\section{CONCLUSION AND Future WORK}

Multirate adaptation is a promising tool in future wireless networks as the corresponding hardware has been available 
off-the-shelf. This paper proposes Multihop Transmission $O P$ portunity (MTOP), which implements relay-oriented, pathcentric frame forwarding mechanism so as to achieve lowlatency, high-throughput communication in wireless multihop networks. Feasibility of MTOP has been proven via analysis and a small-scale testbed based on USRP/ GNU Radio platform. Our performance study based on ns-2 network simulator shows that MTOP performs better than fixed-rate scenarios (6 54Mbps), PRAM, and TXOP in terms of PDR and packet delay in the entire range of node density. This is due to the adaptive behavior of MTOP under different network conditions and the aggressive frame forwarding mechanism in MTOP.

MTOP opens up several interesting directions of research to pursue. First, MTOP can be usefully employed in multiradio/multi-channel networks, typically found in the backhaul of wireless mesh networks. Second, MTOP can also be utilized in WLANs. Allowing multi-hopping at each AP-based network is useful to increase he coverage at no additional cost for deployment of infrastructures.

\section{ACKNOWLEDGMENT}

The work described in this paper was supported in part by the NSF under Grant CNS-0831853, CNS-0821319 and CNS-0721529, and NRF (Korea) WCU Grant R31-2008-00010100-0.

\section{REFERENCES}

[1] IEEE 802.11-1999, Part 11: Wireless LAN Medium Access Control (MAC) and Physical Layer (PHY) Specifications, IEEE Std., 1999.

[2] I. F. Akyildiz, X. Wang, and W. Wang, "Wireless mesh networks: a survey," Elsevier Computer Networks, vol. 47, no. 4, pp. 445-487, Mar. 2005.

[3] J. Bicket, D. Aguayo, S. Biswas, and R. Morris, "Architecture and evaluation of an unplanned 802.11b mesh network," in Proc. of ACM MobiCom, Aug.-Sept. 2005, pp. 31-42.

[4] P. Hui, J. Crowcroft, and E. Yoneki, "Bubble rap: social-based forwarding in delay tolerant networks," in Proc. of ACM MobiHoc, May 2008, pp. 241-250.

[5] H. Rheingold, Smart mobs: the next social revolution. Cambridge, Perseus Book Group, 2002

[6] K. Seada and C. Perkins, "Social networks: the killer app for wireless ad hoc networks?" Nokia, Tech. Rep. NRC-TR-2006-010, Aug. 2006.

[7] A. Akella, G. Judd, S. Seshan, and P. Steenkiste, "Self-management in chaotic wireless deployments," in Proc. of ACM MobiCom, Aug.-Sept. 2005 , pp. $185-199$.

[8] IEEE 802.11e-2005, Part 11: Wireless Medium Access Control (MAC) and Physical Layer (PHY) Specifications: Medium Access Control (MAC) Enhancements for Quality of Service (QoS), IEEE Std., 2005.

[9] B. Sadeghi, V. Kanodia, A. Sabharwal, and E. Knightly, "Opportunistic media access for multirate ad hoc networks," in Proc. of ACM MobiCom, Sept. 2002, pp. 24-35.

[10] G. Tan and J. Guttag, "Time-based fairness improves performance in multi-rate WLANs," in Proc. of The USENIX Annual Technical Conference, 2004.

[11] I. Tinnirello and S. Choi, "Temporal fairness provisioning in multi-rate contention-based 802.11e WLANs," in Proc. of IEEE WoWMoM, June 2005 , pp. 220-230.

[12] Universal Software Radio Platform (USRP), http://www.ettus.com/.

[13] GNU Radio Project, http://www.gnuradio.org/trac/.

[14] The Network Simulator NS-2, http://www.isi.edu/nsnam/ns/.

[15] S. Kang, C. Yu, C. R. Das, and G. Cao, "Path-centric on-demand rate adaptation for mobile ad hoc networks," in Proc. of IEEE ICCCN, Aug. 2009, pp. 1-6.

[16] C. E. Perkins and E. M. Royer, "Ad-hoc on-demand distance vector routing," in Proc. of IEEE Workshop on Mobile Computing Systems and Applications, vol. 2, 1999, pp. 90-100.
[17] M. Heusse, F. Rousseau, G. Berger-Sabbatel, and A. Duda, "Performance anomaly of $802.11 \mathrm{~b}$," in Proc. of IEEE INFOCOM, vol. 2, Mar.Apr. 2003, pp. 836-843.

[18] IEEE 802.15.2-2003, Part 15.2: Coexistence of Wireless Personal Area Networks with Other Wireless Devices Operating in Unlicensed Frequency Bands, IEEE Std., 2003.

[19] Orinoco 11b Client PC Card Specification, Available from http://www. proxim.com/learn/library/datasheets/11bpccard.pdf, 2003.

[20] K. Marquess, "Physical model sub-group discussion and questions," IEEE 802.15/138R0, 1999.

[21] C. Yu, K. G. Shin, and L. Song, "Link-layer salvaging for making routing progress in mobile ad hoc networks," in Proc. of ACM MobiHoc, May 2005, pp. 242-254.

[22] M. Bertocco, G. Gamba, and A. Sona, "Experimental optimization of CCA thresholds in wireless sensor networks in the presence of interference," in Proc. of IEEE EMC Europe, 2007.

[23] R. Gummadi, D. Wetherall, B. Greenstein, and S. Seshan, "Understanding and mitigating the impact of RF interference on 802.11 networks," in Proc. of ACM SIGCOMM, Aug. 2007, pp. 385-396.

[24] S. Narayanaswamy, V. Kawadia, R. S. Sreenivas, and P. R. Kumar, "Power control in ad-hoc networks: theory, architecture, algorithm and implementation of the COMPOW protocol," in Proc. of European Wireless Conference, Feb. 2002.

[25] H. Zhai and Y. Fang, "Physical carrier sensing and spatial reuse in multirate and multihop wireless ad hoc networks," in Proc. of IEEE INFOCOM, Apr. 2006, pp. 1-12.

[26] C. E. Perkins and P. Bhagwat, "Highly dynamic destination-sequenced distance-vector routing (DSDV) for mobile computers," ACM SIGCOMM Computer Communication Review, vol. 24, no. 4, pp. 234-244, 1994.

[27] G. Holland, N. Vaidya, and P. Bahl, "A rate-adaptive MAC protocol for multi-hop wireless networks," in Proc. of ACM MobiCom, July 2001, pp. 236-251.

[28] T.-Y. Lin and J. C. Hou, "Interplay of spatial reuse and SINR-determined data rates in CSMA/CA-based, multi-hop, multi-rate wireless networks," in Proc. of IEEE INFOCOM, May 2007, pp. 803-811.

[29] A. Jow, C. Schurgers, and D. Palmer, "CalRadio: a portable, flexible 802.11 wireless research platform," in Proc. of ACM MobiEval, June 2007, pp. 49-54.

[30] K. A. Jamieson, "The SoftPHY abstraction: from packets to symbols in wireless network design," Ph.D. dissertation, MIT, June 2008.

[31] GNU Radio Source, http://gnuradio.org/trac/browser/gnuradio/trunk/ usrp/fpga/sdr_lib/adc interface.v.

[32] J. Lee, W. Kim, S.-J. Lee, D. Jo, J. Ryu, T. Kwon, and Y. Choi, "An experimental study on the capture effect in 802.11a networks," in Proc. of ACM WinTECH, Sept. 2007, pp. 19-26.

[33] BBN ADROIT Project, http://acert.ir.bbn.com/projects/adroit/

[34] R. Dhar, G. George, A. Malani, and P. Steenkiste, "Supporting integrated MAC and PHY software development for the USRP SDR," in Proc. of IEEE Workshop on Networking Technologies for Software Defined Radio (SDR) Networks, 2006.

[35] T. Schmid, O. Sekkat, and M. B. Srivastava, "An experimental study of network performance impact of increased latency in software defined radios," in Proc. of ACM WinTECH, Sept. 2007, pp. 59-66.

[36] D. Johnson and D. Maltz, Dynamic Source Routing in Ad-Hoc Wireless Networks. Ch. 5, Mobile Computing, edited by T. Imielinski, H. Korth. Kluwer Academic Publishers, 1996.

[37] J. Broch, D. A. Maltz, D. B. Johnson, Y.-C. Hu, and J. Jetcheva, "A performance comparison of multi-hop wireless ad hoc network routing protocols," in Proc. of ACM MobiCom, Oct. 1998, pp. 85-97.

[38] A. Kamerman and L. Monteban, "WaveLAN-II: a high-performance wireless LAN for the unlicensed band," Bell Labs Technical Journal, vol. 2, no. 3, pp. 118-133, Summer 1997.

[39] B. Awerbuch, D. Holmer, and H. Rubens, "High throughput route selection in multi-rate ad hoc wireless networks," Johns Hopkins University, Tech. Rep., 2004.

[40] R. Draves, J. Padhye, and B. Zill, "Routing in multi-radio, multi-hop wireless mesh networks," in Proc. of ACM MobiCom, Sept.-Oct. 2004 pp. 114-128.

[41] S. H. Y. Wong, H. Yang, S. Lu, and V. Bharghavan, "Robust rate adaptation for 802.11 wireless networks," in Proc. of ACM MobiCom, Sept. 2006, pp. 146-157. 